\title{
Validation of the Arabic linguistic version of the International Consultation on Incontinence Questionnaire-overactive bladder (ICIQ-OAB)
}

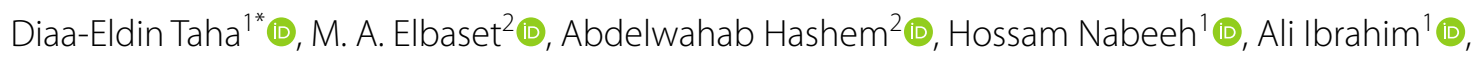
Megan N. Pardoe 3 and Ahmed S. Elhefnawy²

\begin{abstract}
Background: Overactive bladder $(\mathrm{OAB})$ is a health burden that needs an Arabic assessment tool. The idea is to validate the Arabic version of the International Consultation on Incontinence Questionnaire-overactive bladder (ICIQ-OAB).
\end{abstract}

Methods: A multicenter cross-sectional study carried out between March 2019 and February 2020. The translated ICIQ-OAB was used to assess the 227 patients. The enrolled patients were 112 complaining of symptoms suggestive of OAB, 115 healthy without LUTS symptoms. Additionally, patients with and without BOO symptoms were included. The reliability assessment of the internal consistency was done using Cronbach's a test. With the aid of Spearman's correlation coefficient $(r)$, the interdomain associations were assessed. The Mann-Whitney test was used to assess the discrimination validity.

Results: A high internal consistency between the mean scores of women with and those without OAB as well as BOO groups, Cronbach's alpha value was 0.82. A strong correlation obviates among whole ICIQ domains in OAB set $(P \leq 0.001)$. Equally, a high correlation exists among each domain in the BOO group, and Cronbach's alpha value was 0.82. In comparison with control, highly significant scores exist for all ICIQ-OAB domains and entire points in the BOO as well as OAB sets $(P<0.001)$. The ICIQ-OAB was found to have good discriminant validity.

Conclusion: A formulated and approved ICIQ-OAB -Arabic release is a value tool for addressing OAB symptom complex. The easy questionnaire will be a useful tool in grading the bother symptoms in Arabic speaking inhabitant.

Keywords: International Consultation on Incontinence Questionnaire (ICIQ-OAB); Overactive bladder, Arabic Version, Validation Studies

\section{Background}

Overactive bladder (OAB) is a group of symptoms with or without frequent urinary incontinence in the absence of an infection or other obvious pathologies, often with

\footnotetext{
*Correspondence: drdiaaeldin@gmail.com

${ }^{1}$ Department of Urology, Faculty of Medicine, Kafrelsheikh University, Kafrelsheikh, Egypt

Full list of author information is available at the end of the article
}

complaints of frequent urination and urinary urgency (day and night) along with or without urge incontinence [1]. The urgency incontinence exerts a profound adverse negativity on quality of life (QOL) $[2,3]$. The overall prevalence was between 11.8 and $16.9 \%$ [4].

The self-accomplished questionnaires are the more than valued tool for weighting the bother of LUTS [5]. A questionnaire titled the International Consultation on Incontinence Questionnaire (ICIQ-OAB) that was 
primarily used in English was purchased to obviate the bother symptoms linked with OAB in a single score [6]. It was translated and validated in different languages [7-9].

ICIQ-OAB involves urination daytime frequency (ICIQ-OAB -3), nocturia frequency (ICIQ-OAB -4), urinary urgency frequency (ICIQ-OAB -5), and urgency incontinence (ICIQ-OAB -6). The total score entails the sum of the entire symptoms. The intensity of these symptoms was graded as $0-4$. The more the score rise, the more intense will be the bother symptoms. With this obvious questionnaire, patients can be evaluated more precisely.

On the one hand, the short form of OAB questionnaire can be used by general practitioners and clinicians in primary and secondary clinics for OAB patients screening. On the other hand, it provides a suitable mean for performing research projects $[6,10]$.

Arabic is spoken by as many as 420 million people (native and non-native) all over the world. The Arabic validation of the ICIQ-OAB questionnaire was not provided.

The statue intend of current study is to show to what extent was the ICIQ-OAB is valid and reliable.

\section{Methods}

This multicenter study is a cross-sectional one which was conducted between March 2019 and January 2020, in two tertiary centers, $x x x x x x x$ University, urology department and Urology and nephrology center, xxxxxx University. The ethical committee approval, number 970/2018, was obtained. All participants consented the questionnaire. The study was fashioned in pursuance with the declaration of Helsinki.

The owners of ICIQ-OAB give the permission to validate. The authors were permitted to translate a version in English language of ICIQ-OAB (source) into the Arabic one. ICIQ-OAB Arabic validation was executed via the following steps. Initially, two autonomous professional native Arabic translators, who can speak English appropriately, translated the questionnaire from English to Arabic. Both the translators and urologists (DE and $\mathrm{AH})$ rechecked the translation. Then after, the translated text was acceptably comprehensive to patients at various schooling levels. Thirdly, the draft text, Arabic version, was back translated into English executed by two independent, outstanding translators. Checking of the back translations was done and some slight corrections were made. Some Arabic grammars and spelling was revised to finally have the second draft. Eventually, a pilot audition was carried on thirty patients who have OAB.

The changes ultimately were done, the English translation form as well as native release of the Arabic ICIQ$\mathrm{OAB}$ was sent to the former composer (ICIQ advisory board) for proper comparison. This ultimate release was utilized for the entire validation process.

A pilot audition was carried out to appraise the questionnaire applicability; 40 (of which 20 were female) patients who had OAB symptoms were interviewed. Interviewed cohort declared each question clear as well as comprehensive. No obstacles were perceived in answering it, and no more changes were urged.

The study entailed two groups. The preliminary entailed 112 with symptoms suggestive of $\mathrm{OAB}(\mathrm{OAB}$ group), another comparative group without LUTS, who concurred to finalize the questionnaire, was the control group.

We excluded patients who have active UTIs, mixed urinary incontinence, pelvic pain syndrome, and/or who are concurrently on medications that might affect the lower tract.

The Arabic ICIQ-OAB version was finished at baseline and after two weeks, on the contrary, the controls just completed it once.

A test-retest reliability and internal consistency was assessed. Test-retest reliability detects the concurrence strength between the double time intervals, the first and the second questionnaires. The intraclass correlation coefficient (ICC) was computed for the overall score. Moreover, separate item was measured by kappa coefficient.

Explanation of the correlation coefficient was utilized according to the proposal by Cohen; weak correlation coefficient was 0.1 , while 0.3 moderate, and 0.5 strong.

\subsection{Sample size calculation}

Putting in consideration the statistical error, the expected difference among groups regarding total ICIQ-OAB was 0.6 [7]. The required sample size that achieves a higher difference was around 90 in every arm.

\subsection{Statistical analysis}

$\mathrm{IBM}^{\circledR}$ version 21 was used for statistical analysis. Significance level was set to $p<0.05$. Cronbach's $\alpha$ test was utilized to test the reliability of internal consistency, while Wilcoxon signed rank test was used to show the test-retest reliability. Moreover, Spearman correlation assessed the concurrent external validity. Values more than 0.70 were proposed to attain reasonable consistency and reliability.

\section{Results}

Of all, 227 involvers were enrolled in the current study. We excluded thirty seven who did not fit the proposed inclusion parameters.

The Arabic translation reliability of test-retest was adequate. For separate symptom score, the range of kappa 
Table 1 Internal consistency (Cronbach's a) and interdomain association by Spearman's correlation coefficient in the OAB group

\begin{tabular}{llllll}
\hline Domain & Cronbach's & $\begin{array}{l}\text { Frequency } \\
r(p)\end{array}$ & $\begin{array}{l}\text { Nocturia } \\
r(p)\end{array}$ & $\begin{array}{l}\text { Urgency } \\
r(p)\end{array}$ & $\begin{array}{l}\text { UUI } \\
(p)\end{array}$ \\
\hline Frequency & 0.82 & & $0.485(\leq 0.001)$ & $0.595(\leq 0.001)$ & $0.482(\leq 0.001)$ \\
Nocturia & 0.85 & $0.458(\leq 0.001)$ & & $0.647(\leq 0.001)$ & $0.572(\leq 0.001)$ \\
Urgency & 0.83 & $0.559(\leq 0.001)$ & $0.647(\leq 0.001)$ & & $0.787(\leq 0.001)$ \\
UUI* & 0.82 & $0.482(\leq 0.001)$ & $0.572(\leq 0.001)$ & $0.787(\leq 0.001)$ & \\
\hline
\end{tabular}

*UUl urge urinary incontinence

Table 2 Discrimination properties of the Arabic ICIQ between patients with $\mathrm{OAB}$ and controls

\begin{tabular}{llll}
\hline ICIQ (median, range) & Patients $(n=112)$ & Control $(n=115)$ & $P$ \\
\hline Daytime frequency & $1(0-2)$ & $0(0-1)$ & $\leq 0.000$ \\
Nocturia & $4(0-4)$ & $1(0-2)$ & $\leq 0.000$ \\
Urgency & $4(2-5)$ & $0(0-3)$ & $\leq 0.000$ \\
UUI * & $4(3-5)$ & $1(0-1)$ & $\leq 0.000$ \\
Total ICIQ & $10(8-13)$ & $2(0-3)$ & $\leq 0.000$
\end{tabular}

*UUI urge urinary incontinence

coefficients was from 0.48 to 0.78 , while overall symptom coefficient was 0.710 (95\% CI, 0.48-0.73). Moreover, the Cronbach's alpha value was 0.82 (Table 1 ).

Among the four domains, a high internal consistency was perceived. As regard the OAB groups, based on Cronbach's $\alpha$ test, a higher internal consistency reached. The correlation $(r)$ among frequency and nocturia, urgency, and UUI was $0.68,0.83$, and 0.71 , respectively $(P<0.001)$. Additionally, the correlation $(r)$ among nocturia and urgency, UUI was 0.52 and 0.72 , respectively $(P<0.01)$, Moreover, a low significant correlation exists between urgency and UUI was $(r=0.54(P<0.001)$ (Table 1).

Likewise, inside $\mathrm{BOO}$ set, stronger significance exists. The correlation between frequency and nocturia, urgency, and UUI was $(P<0.001, P=0.003$, and $P<0.001$, respectively), between nocturia and UUI $(P<0.001)$, and between urgency and UUI $(P<0.001)$ (Table 3$)$.

Significant scores in overall domains and entire scores were present between $\mathrm{OAB}$ and control, similarly between BOO set and $(P<0.001)$ (Tables 2,4$)$. This denotes discriminative validity potential.

\section{Discussion}

Questionnaires can help the physicians to assess bother symptoms and eventually postulate treatment plans.

The ICIQ-OAB evolved by the Bristol Urological Institute, based at North Bristol NHS Trust, Bristol [6], validated in different languages [7-9, 11].

The overall score entails the aggregate of all symptom scores that interpret daytime voiding frequency, nighttime voiding frequency, urgency, and UUI.

As a concern, $47 \%$ of patients, who have BOO, might complaint about $\mathrm{OAB}$ symptoms. Moreover, the degree of symptom burden correlated equally with the $\mathrm{BOO}$ degree as well as $\mathrm{OAB}$ at baseline and after treatment [12].

The Arabic translation of the ICIQ-OAB precludes average content validity and internal consistency, was

Table 4 Discrimination properties of the Arabic ICIQ between patients with $\mathrm{BOO}$ with $\mathrm{OAB}-$ like symptoms (BOO group) and controls

\begin{tabular}{llll}
\hline ICIQ ( median, range) & Patients $(n=50)$ & Control $(n=50)$ & $P^{*}$ \\
\hline Daytime frequency & $1(0-2)$ & $0(0-1)$ & $\leq 0.000$ \\
Nocturia & $2(0-3)$ & $1(0-2)$ & $\leq 0.000$ \\
Urgency & $2(0-4)$ & $0(0-3)$ & $\leq 0.000$ \\
UUI & $2(0-4)$ & $1(0-1)$ & $\leq 0.000$ \\
Total ICIQ & $8(6-12)$ & $2(0-3)$ & $\leq 0.000$ \\
\hline
\end{tabular}

* UUl urge urinary incontinence

Table 3 Internal consistency (Cronbach's a) and interdomain association by Spearman's correlation coefficient in the BPH group

\begin{tabular}{llllll}
\hline Domain & Cronbach's & $\begin{array}{l}\text { Frequency } \\
r(p)\end{array}$ & $\begin{array}{l}\text { Nocturia } \\
r(p)\end{array}$ & $\begin{array}{l}\text { Urgency } \\
r(p)\end{array}$ & $\begin{array}{l}\text { UUI } \\
r(p)\end{array}$ \\
\hline Frequency & 0.69 & & $0.457(\leq 0.001)$ & $0.271(\leq 0.001)$ & $0.157(\leq 0.001)$ \\
Nocturia & 0.70 & $0.457(\leq 0.001)$ & & $0.487(\leq 0.001)$ & $0.283(\leq 0.001)$ \\
Urgency & 0.72 & $0.271(\leq 0.001)$ & $0.487(\leq 0.001)$ & & $0.585(\leq 0.001)$ \\
UUI* & 0.69 & $0.157(\leq 0.001)$ & $0.283(\leq 0.001)$ & $0.585(\leq 0.001)$ & \\
\hline
\end{tabular}


stable, and had good construct validity and responsiveness in women with $\mathrm{OAB}$, especially urinary incontinence and/or urgency, and is therefore suitable for the assessment of these symptoms, before and after treatment, in Arabic-speaking women.

The reliability of the Arabic version was proven by the high internal consistency, also an accepted interdomain correlation in both the $\mathrm{OAB}$ and $\mathrm{BOO}$ sets (Tables 1, 3 ), the same that was perceived in other ICIQ-OAB language validation, e.g., portuges, dealing with $\mathrm{OAB}$ [9].

An obvious discrimination validity was perceived by the significant variance in entire scores between diseased and healthy persons (Tables 2,4 ). The high discriminatory power as well as reasonable psychometric properties of the original English-validated questionnaire is the core of this discriminative validity, Moreover, the to and fro translation validation process is considered a value standard for linguistic validation.

Consequently, the current study obviates that the Arabic validated release of the ICIQ-OAB can be easily utilized by urologists to ease and quick patients assessment. Additionally, it is of value that the ICIQ-OAB will aid the grading of $\mathrm{OAB}$ symptoms equally in young as well as geriatric demographics.

To the best of our knowledge, the present study boost come from being elucidated in two tertiary specialized urology clinics. Moreover, equally the subject and the expert evaluated each question separately that reinforce the study. Another strength was the broad diversity of inhabitants, equally males as well as females, with the symptoms.

What limits our study that, some of our patients are not well educated that probably impact their ability to finalize such questionnaires. Another drawback was the incapacity to assess test-retest reliability, for the same reason of low educational levels in various patients.

\section{Conclusion}

A formulated and approved ICIQ-OAB -Arabic release is a value tool for addressing OAB symptom complex. The easy questionnaire will be a useful tool in grading the bother symptoms in Arabic speaking inhabitant.

\section{Abbreviations \\ ICIQ-OAB: International Consultation on Incontinence Questionnaire-overac- tive bladder; OAB: Overactive bladder; BOO: Bladder outlet obstruction; LUTS: Lower urinary tract symptoms; UUI: Urge urinary incontinence.}

\section{Acknowledgements}

None.

\section{Authors' contributions}

DT has finished article writing, MAE collected data collection and revised the article, $\mathrm{AH}$ revised the article, $\mathrm{HN}$ has collected the data and statistical analysis,
Al collected. The data, MNP processed the translation revision, and ASE finialize the article. All authors have read and approved the manuscript.

Funding

None.

Availability of data and materials

Available.

\section{Declarations}

\section{Ethics approval and consent to participate}

The ethical approval committee in faculty of medicine, Mansoura university, was the assigned committee for approving the study. The ethical committee approval number 970/2018, was obtained. All participants consented the questionnaire.

\section{Committee's reference number}

Not applicable.

\section{Consent for publication}

The consent obtained from study participants was verbal. The reason for that is the study doesn't involve intervention or experiment, it just filling a questionnaire, this was approved by the ethics committee as well.The ethical approval committee in faculty of medicine, Mansoura university, was the assigned committee for approving the study.

\section{Competing interests}

All authors declare no conflict of interest.

\section{Author details}

${ }^{1}$ Department of Urology, Faculty of Medicine, Kafrelsheikh University, Kafrelsheikh, Egypt. ${ }^{2}$ Urology and Nephrology Center, Mansoura University, Mansoura, Egypt. ${ }^{3}$ Bristol Urological Institute, 3rd Floor Learning and Research, Southmead Hospital, Bristol BS10 5NB, UK.

Received: 5 February 2021 Accepted: 10 March 2021

Published online: 23 March 2021

\section{References}

1. Abrams P, Cardozo L, Fall M, Griffiths D, Rosier P, Ulmsten U et al (2003) The standardisation of terminology in lower urinary tract function: report from the standardisation sub-committee of the International Continence Society. Urology 61(1):37-49

2. Coyne K, Revicki D, Hunt T, Corey R, Stewart W, Bentkover J et al (2002) Psychometric validation of an overactive bladder symptom and health-related quality of life questionnaire: the OAB-q. Qual Life Res 11(6):563-574

3. Abrams P, Kelleher CJ, Kerr LA, Rogers RG (2000) Overactive bladder significantly affects quality of life. Am J Manag Care 6(11 Suppl):S580-S590

4. W F Stewart 1, J B Van Rooyen, G W Cundiff, P Abrams, A R Herzog, R Corey, et al., Prevalence and burden of overactive bladder in the United States. World J Urol, 2003. 20(6): p. 327-36

5. Dennis Revicki 1, Ron D Hays, David Cella, Jeff Sloan. Recommended methods for determining responsiveness and minimally important differences for patient-reported outcomes. J Clin Epidemiol, 2008. 61(2): p. 102-9

6. International Consultation on Incontinence Modular Questionnaire. Validation Protocol. [Cited 20 Dec 2013]. Available from URL: http://www. iciq.net/validationprotocol.htm

7. Gayan de Silva, Rameez Furukan 2, Malik Goonewardene . Validation of the Sinhala translation of the International Consultation on Incontinence Modular Questionnaire for female lower urinary tract symptoms among women in Sri Lanka. International urogynecology journal, 2017. 28(12): 1895-9

8. Ekanayake, Chanil D.Pathmeswaran, Arunasalam Nishad, A. A. Nilanga Samaranayake, Kanishka U.Wijesinghe, et al., Validation of the International Consultation on Incontinence Questionnaire-Vaginal Symptoms (ICIQ-VS) in 
two south-Asian languages. International urogynecology journal, 2017. 28(12): p. 1849-55

9. Simone Botelho Pereira, Rosane do Rocio Cordeiro Thiel, Cássio Riccetto, Joseane Marques da Silva, Larissa Carvalho Pereira, Viviane Herrmann, et al., Validation of the International Consultation on Incontinence Questionnaire Overactive Bladder (ICIQ-OAB) for Portuguese. Revista brasileira de ginecologia e obstetricia : revista da Federacao Brasileira das Sociedades de Ginecologia e Obstetricia, 2010. 32(6): 273-8

10. Catherine Acquadro, Zoe Kopp, Karin S Coyne, Jacques Corcos, Andrea Tubaro, Myung-Soo Choo, et al., Translating overactive bladder questionnaires in 14 languages. Urology, 2006. 67(3): p. 536-40
11. Chanil D Ekanayake, Arunasalam Pathmeswaran, A A Nilanga Nishad, Kanishka U Samaranayake, Prasantha SWijesinghe. Translation and validation of ICIQ-FLUTS for Tamil-speaking women. International urogynecology journal, 2017. 28(12): 1875-81

12. Akira Tsujimura 1, Tetsuya Takao, Yasushi Miyagawa, Hidenobu Okuda, Keisuke Yamamoto, Shinichiro Fukuhara, et al., Survey of overactive bladder symptoms influencing bother before and after treatment with tamsulosin hydrochloride in Japanese patients with benign prostatic hyperplasia. Urology, 2011. 78(5): 1058-62

\section{Submit your manuscript to a SpringerOpen ${ }^{\circ}$ journal and benefit from:}

- Convenient online submission

- Rigorous peer review

- Open access: articles freely available online

- High visibility within the field

- Retaining the copyright to your article

Submit your next manuscript at $\boldsymbol{\nabla}$ springeropen.com 